\title{
Editorial zur Umbenennung in Z'Flucht: Zeitschrift für Flucht- und Flüchtlingsforschung
}

In den vergangenen Jahren hat sich die Forschung in Deutschland über diverse Aspekte des weltweiten Fluchtgeschehens und insbesondere über die Aufnahme bzw. Nicht-Aufnahme von Schutzsuchenden in Deutschland und Europa erheblich intensiviert. Dies verdeutlichen auch die Beiträge, die in den beiden ersten Jahrgängen der Z'Flucht erschienen sind. Ausmachen lässt sich außerdem, dass Debatten über die Benennung des Forschungsfeldes und damit über dessen Selbstverständnis einsetzten: Ist der Begriff der >Flüchtlingsforschungく (weiterhin) angemessen? Vermag jener der `Fluchtforschung ihn zu ersetzen? Braucht es einen anderen Begriff, der wie die >Forced Migration Studies` eine Anlehnung an die Migrationsforschung bietet?

Im Oktober 2018 beschlossen die Mitglieder des damaligen >Netzwerks Flüchtlingsforschung «, das Kooperationspartner der Z'Flucht ist, eine Umbenennung in das `Netzwerk Fluchtforschung . Dieser Begriff beschreibe den Gegenstand des Forschungsfeldes eindeutiger, vor allem sei er inklusiver. Für eine Zeitschrift, die sich als Plattform der Auseinandersetzung mit Ergebnissen und Perspektiven dieser Forschungsrichtung versteht, sind solche Überlegungen von höchster Bedeutung. Die Herausgeberin und die Herausgeber haben sich vor dem Hintergrund der Debatten um die Bezeichnung des Netzwerkes dazu entschlossen, die Z'Flucht umzubenennen. Wir haben allerdings einen anderen Namen als das Netzwerk gewählt, die >Flüchtlingsforschung` im Titel beibehalten und um die >Fluchtforschungく ergänzt.

Das vorliegende Editorial zielt darauf, die Entscheidung für den neuen Doppelnamen der >Zeitschrift für Flucht- und Flüchtlingsforschungく kurz zu erläutern. Es möchte außerdem weiterführende Diskussionen anregen und dazu einladen, kritische Beiträge zu Fragen der Konzeptualisierung der Flucht- und Flüchtlingsforschung einzureichen. Mit der Umbenennung wollen wir sicherstellen, dass eine Vielfalt der Themen sowie disziplinärer, methodologischer und methodischer Zugänge gewahrt werden kann und die Zeitschrift auch weiterhin ihrer Funktion als Plattform für alle interessierten Wissenschaftler*innen und Praktiker*innen in dem Feld nachzukommen vermag. 


\section{Flüchtlingsforschung?}

Ohne einen Bezug auf die internationale Debatte lässt sich eine Diskussion über die Bezeichnung des Forschungsfeldes gewiss nicht führen. Die englischsprachige Forschung diskutiert bekanntlich seit den späten 1990er Jahren, ob sie sich als >Refugee Studies` oder als >Forced Migration Studies` versteht. Da die Z'Flucht einen »Anknüpfungspunkt für das internationale Feld der Forced Migration and Refugee Studies« bieten möchte (Berlinghoff et al. 2017: 4), ist die Berücksichtigung der dortigen Abwägungen bedeutsam.

Einige Forscher*innen verweisen beispielsweise darauf, dass sie einen seit langem etablierten Rechtsbegriff meinen, wenn sie von >Refugees sprechen (vgl. Hathaway 2007). Wegen der ihnen zugewiesenen spezifischen Rechte, die als ein hohes Gut verstanden werden müssten, seien sie eindeutig von anderen Migrant*innen zu unterscheiden. Andere wenden gegen die Nutzung des Begriffs ein, es handele sich um eine staatliche Ordnungskategorie. Deren Verwendung verenge die Beobachtungsperspektive einer vornehmlich sozialwissenschaftlich betriebenen Forschung, die ein soziales Phänomen reflexiv und ohne die Leitplanken einer Statusfrage zu thematisieren habe (vgl. DeWind 2007). Scott FitzGerald und Rawan Arar (2018) verwiesen kürzlich auf einen Weg zur Inklusion beider Auffassungen. Sie hegen keinen Zweifel daran, dass es sich um ein einheitliches Forschungsfeld handelt, identifizieren aber zwei Schulen, die sich aus ihrer Sicht ergänzen: Ein realistischer Ansatz untersuche etwa die Wirkungsmacht der Flüchtlingskategorie in Flüchtlingsrecht und -politik. Ein konstruktivistischer Ansatz fokussiere hingegen auf das Labeling sowie die Veränderbarkeit solcher Kategorien und betone das Relationale und Prozesshafte von Flucht, beispielsweise im Kontext gemischter Migrationsbewegungen.

Wie die Bezugnahme auf den Begriff des $>$ Refugee $<$ wird auch jene auf den 〉Flüchtling « in der deutschsprachigen Forschungslandschaft teilweise kritisch gesehen. Weil es sich nicht um eine wissenschaftliche, sondern um eine rechtliche Kategorie handele, bleibe er politisch umkämpft (Friese 2017). Außerdem sei der Begriff negativ konnotiert, nicht zuletzt aufgrund der herabsetzenden Endung auf >lingく. Zwar wies auch Hannah Arendt (1943) die Bezeichnung >Refugee` zurück, weil er auf eine implizit defizitäre Person verweise. Dabei ist aber gerade die Beobachtung der Auswirkungen eines minderen Status einer Person, die nicht über das Recht verfüge, Rechte zu haben, wie Arendt (2004 (1951)) später formulierte, für die Geistes-, Kultur- und Sozialwissenschaften von besonderer Relevanz. Spätestens mit der Genfer Flüchtlingskonvention von 1951 und deren globaler Durchsetzung ist >Flüchtling « weit mehr als ein Pejorativum. So kann der 
Begriff als juristischer Status und politische Kategorie in administrativer wie auch sozialer Praxis nicht aufgegeben werden, ohne zugleich die Rechte und die spezifischen gesellschaftlichen Positionen der so kategorisierten Personen zu ignorieren.

>Flüchtlingsforschungく will mithin die Anwendung von Kategorien und die Folgen von Kategorisierungen untersuchen. Flüchtlinge versteht sie also nicht notwendigerweise als spezifische soziale Gruppe oder als Individuen. Sie erforscht vielmehr das Aushandeln und das Setzen von Kategorien, die An- und Aberkennung rechtlicher Privilegien wie auch deren Auswirkungen auf die als Flüchtlinge oder andere Statusgruppen politisch und rechtlich definierten Menschen. Sie widmet sich daher unter anderem dem Konflikt um Status und um Rechte zwischen Staaten und Schutzsuchenden, zum Beispiel in Bezug auf Menschenrechte und (Staats-)Bürgerschaft. Darüber hinaus werden Zuschreibungen etwa im administrativen Labeling in den Blick genommen (Zetter 1991) und mit Rollen und Selbstverständnissen Geflüchteter verknüpft.

\section{Fluchtforschung?}

Die englischsprachige Forced Migration and Refugee Studies wie auch die deutschsprachige Forschung in diesem Feld widmen sich Fragen des Schutzes von Zufluchtsuchenden, der Folgen von Gewährung oder Nicht-Gewährung von Aufnahme und Schutz sowie der Handlungsmacht von Menschen in Aufnahmesituationen. Demgegenüber steht die Frage zurück, warum sich Menschen in welchen Konstellationen, unter welchen Umständen und mit welchen Konsequenzen in Bewegung gesetzt haben bzw. setzen mussten. Auch die >Fluchtく selbst, also der Prozess des Bewegens, die auf die Suche nach einem sicheren Ort ausgerichtet ist, wird weniger intensiv untersucht (Davenport et al. 2003: 29-30; BenEzer/ Zetter 2015). >Fluchtforschung hingegen nimmt sich explizit vor, die Hintergründe der durch Gewalt induzierten Bewegungen und die Mobilitätsmuster, die häufig durch ein Kontinuum der Gewalt gekennzeichnet sind, zu untersuchen. Durch den Bezug auf gewaltinduzierte Bewegungen verweist der Begriff `Fluchtく auch auf Phänomene, die wie Vertreibungen, Umsiedlungen, Deportationen, Evakuierungen oder Verschleppungen als Ergebnis der Androhung oder Anwendung von Gewalt zu verstehen sind.

Mit der Verwendung des Begriffs der Fluchtforschung setzen wir uns von dem in Deutschland durchaus verbreiteten Konzept der `Zwangsmigrationsforschung< $\mathrm{ab}$ - nicht in erster Linie deshalb, weil es sich um einen sehr sperrigen Begriff 
handelt, sondern vor allem weil der Begriff des \Zwangs` in vieler Hinsicht uneindeutig ist. Auch zeigen zahlreiche Debatten in der Forschung, dass die häufig verwendete Gegenüberstellung von >freiwilliger` und >unfreiwilliger Bewegung nicht tragfähig ist (Düvell 2006: 18).

Der Begriff der >Flucht $\iota$, der keine direkte Entsprechung im englischen wissenschaftlichen Diskurs hat, knüpft an die deutschsprachige Forschungstradition zu Flucht und Vertreibung an. >Fluchtforschung« steht dabei keineswegs in einem Gegensatz zur >Flüchtlingsforschung`. Ihre Beobachtungsfelder überlappen sich und bieten komplementäre Zugänge zu einem multiperspektivischen Forschungsfeld.

\section{Programmatik der `Zeitschrift für Flucht- und Flüchtlingsforschung،}

Die Umbenennung dieses Periodikums in `Z'Flucht: Zeitschrift für Flucht- und Flüchtlingsforschung` ist Ausdruck des Versuchs, gesellschaftliche und wissenschaftliche Debatten um das Selbstverständnis des Forschungsfeldes aufzugreifen und damit zu einem multidisziplinären Verständnis der komplexen Themen Flucht und Flüchtlinge beizutragen. Daher soll die Umbenennung der Zeitschrift Debatten um das Selbstverständnis eines sich rasch herausbildenden Forschungsfeldes sowie der Vielfalt der hier vertretenen wissenschaftlichen Ansätze und Perspektiven gerecht werden.

Auch dieser Titel vermag nicht alle Themen und Aspekte des Forschungsfeldes zu erfassen, die in der Z'Flucht ein Forum finden sollen. Die Zeitschrift bietet jedoch explizit Raum für kritische Analysen zu vielfältigen Fragen, die Flucht bzw. Flüchtlinge und andere Schutzsuchende betreffen. Ebenso besteht ausdrücklich die Möglichkeit, in der Z'Flucht Beiträge zu allen Formen und Aspekten gewaltinduzierter Bewegung zu veröffentlichen. Damit zielt die Zeitschrift auf eine langfristige Vernetzung und Kooperation der Forschung insbesondere über konzeptionelle und disziplinäre Grenzen hinweg. So bildet sie ein Forum für ein stets im Wandel begriffenes Forschungsfeld, das auf eine dauernde Auseinandersetzung über Begriffe, Konzeptionen und Methodologien angewiesen ist.

\section{Literatur}

Arendt, Hannah (1943), We Refugees, Menorah Journal, 31 (1), 69-77. Arendt, Hannah (2004 (1951)), The Origins of Totalitarianism, New York. 
BenEzer, Gadi/Zetter, Roger (2015), Searching for Directions: Conceptual and Methodological Challenges in Researching Refugee Journeys, Journal of Refugee Studies, 28 (3), 297-318.

Berlinghoff, Marcel, et al. (2017), Editorial, Zeitschrift für Flüchtlingsforschung, $1(1), 3-8$.

Davenport, Christian A./Moore, Will H./Poe, Steven C. (2003), Sometimes you just have to leave: Domestic Threats and Forced Migration, 1964-1989, International Interactions, 29 (1), 27-55.

DeWind, Josh (2007), Response to Hathaway, Journal of Refugee Studies, 20 (3), 381-385.

Düvell, Frank (2006), Europäische und internationale Migration: Einführung in historische, soziologische und politische Analysen, Münster.

FitzGerald, David Scott/Arar, Rawan (2018), The Sociology of Refugee Migration, Annual Review of Sociology, 44 (1), 387-406.

Friese, Heidrun (2017), Flüchtlinge: Opfer - Bedrohung - Helden. Zur politischen Imagination des Fremden, Bielefeld.

Hathaway, James C. (2007), Forced Migration Studies: Could We Agree Just to >Date<?, Journal of Refugee Studies, 20 (3), 349-369.

Zetter, Roger (1991), Labelling Refugees: Forming and Transforming a Bureaucratic Identity, Journal of Refugee Studies, 4 (1), 39-62. 